\title{
Proportion and Clinical Outcome of Bleeding Manifestations in Confirmed Dengue Fever Cases - A Descriptive Longitudinal Study from Ernakulam, Kerala, India
}

\author{
Amrish Thannickal Thazhath ${ }^{1}$, John Sajith², Jacob K. Jacob³ ${ }^{3}$ George Jilse ${ }^{4}$, Samuel Suma ${ }^{5}$, Thalappally Bhagyanath ${ }^{6}$ \\ 1, 2, 3, 4, 5, 6 Department of Internal Medicine, Government Medical College, Ernakulam, Kerala, India.
}

\section{ABSTRACT}

\section{BACKGROUND}

Dengue fever is expanding in newer areas globally, with greater morbidity and mortality. We wanted to determine the proportion of bleeding manifestations among the confirmed dengue fever patients and evaluate the clinical outcomes in those with bleeding manifestations.

\section{METHODS}

230 Dengue confirmed patients admitted in Government Medical College, Ernakulam from January 2018 to December 2018 were enrolled for the study. Data was recorded and entered in Microsoft Excel, and analysed using SPSS.

\section{RESULTS}

In the current study out of 230 subjects, bleeding manifestations were more common in 21 - $30 \& 41$ - 50 age groups. Out of 230 subjects, 102 had bleeding manifestations (44.3\%) like petechiae (34\%), purpura (24\%), echymosis (19.1\%), gum bleeding (17.8\%), menorrhagia (10.9\%), melena (10.4\%), conjunctival haemorrhage ( $9.1 \%)$, haematuria (4.8\%), epistaxis (3.5\%), haemoptysis (2.2\%) and hematemesis $(0.9 \%) .86$ subjects $(37.4 \%)$ received platelet transfusion on an average of 4 units. Age group, fever, previous history of dengue, IgG positivity, NS1 positivity were significantly $(\mathrm{P}<0.0001)$ associated with bleeding. Most of the bleeding manifestations were significant in patients who had lower platelet counts on the first day of admission when it was less than ten thousand.

\section{CONCLUSIONS}

$44.3 \%$ had bleeding manifestations with significant association between age, fever and IgG positivity. A low platelet count on admission especially less than 10,000 was significantly associated with these bleeding manifestations. These significant observations are important for both the primary care and tertiary care physicians in meticulous management and referral of dengue patients.

\section{KEY WORDS}

Dengue Fever, Bleeding Manifestations, Ns1 Antigen, Platelet Count.

\author{
Corresponding Author: \\ Dr. Jacob K. Jacob, \\ Bethel, Moolepadam, \\ Kalamassery \\ P.O - 683104, Ernakulam, \\ Kerala, India. \\ E-mail: jacobkjacob@yahoo.com \\ DOI: $10.14260 /$ jemds/2021/396
}

How to Cite This Article:

Thazhath AT, Sajith J, Jacob JK, et al.. Proportion and clinical outcome of bleeding manifestations in confirmed dengue fever cases - a descriptive longitudinal study from Ernakulam, Kerala, India. J Evolution Med Dent Sci 2021;10(26):1921-1925, DOI:

Submission 23-01-2021,

Peer Review 20-04-2021,

Acceptance 26-04-2021,

Published 28-06-2021.

Copyright (C) 2021 Amrish Thannickal Thazhath et al.. This is an open access article distributed under Creative Commons Attribution License [Attribution 4.0 International (CC BY 4.0)] 


\section{BACKGROUND}

Dengue is a mosquito-borne viral disease which is endemic in more than 100 countries in Asia, America and Africa, and reoccurs every year. ${ }^{1}$ Being a self-limiting acute mosquito transmitted disease, it is characterized by fever, rash, myalgia, joint pains, headache, nausea and vomiting. The causative agent is an arbovirus which is spread by Aedes mosquito. In some cases it results in Dengue haemorrhagic fever (DHF) or even in its severe form called Dengue Shock Syndrome which can threaten the patient's life that primarily occurs due to increased vascular permeability and shock. ${ }^{2}$ Dengue is a disease with different clinical presentations and often results in unpredictable clinical progression and outcome. ${ }^{3}$

Dengue viruses are the most dangerous human arboviruses identified till date and they are seen in the form of four distinct serotypes (DENV - 1, DENV - 2, DENV - 3 and DENV - 4). The four serotypes of the virus - DENV 1, 2, 3 and 4 - belong to the genus flavivirus and family flaviviridae, and are predominantly transmitted by Aedes aegypti and few other members of aedes species. ${ }^{4}$ Being an arthropod - borne virus, dengue virus is an RNA virus with a high mutation rate ${ }^{1}$ Infection with one of the serotypes does not give immunity against infection with the other serotypes. ${ }^{5}$ It is estimated that almost 2.5 billion people are living at risk with dengue fever, mainly in tropical and subtropical countries as well as nearly $50-100$ million people are infected annually with around 25,000 deaths. ${ }^{6}$

Based on a survey which depicts that out of 390 million dengue infections which occur annually (95\% credible interval 284 - 528 million), nearly 96 million (67 - 136 million) are clinically manifested (with any severity of disease). In accordance with another research about the widespread presence of dengue, it illustrates that 3.9 billion people in 128 nations, are exposed to the danger of getting affected with dengue viruses. ${ }^{7}$ There was acute dengue outbreak in just 9 countries before 1970s but now has been widely spread in more than a hundred countries with the 6 WHO regions except the European Region. Among them the regions such as the Americas, South-East Asia and the Western Pacific are the most critically affected. ${ }^{7}$ Considering the situation in India, dengue is endemic in almost all states and is one of the leading causes of hospitalization. ${ }^{8}$ Dengue fever was first reported in 1956 from Vellore district in Tamil Nadu. Thereafter, the first DHF outbreak occurred in 1963 in Calcutta in West Bengal with almost $30 \%$ of the cases showing hemorrhagic manifestations. All the four serotypes, that is, Dengue 1, 2, 3 and 4 have been isolated in India. ${ }^{2}$

Kerala may still be paying the price for the devastating floods of the year 2018. According to figures available with the National Vector Borne Disease Control Programme (NVBDCP), till 30th September 2018, the total number of dengue cases in India was 40868 with 83 deaths. Of these 42 $\%$ were from Kerala where floods and landslides left a trail of devastation. Though the Kerala death toll was less than 2017, the state witnessed proportionately larger number of deaths in 2018 compared to 2017. Last year only $11 \%$ dengue deaths occurred in Kerala. It is also important to note that Kerala, a southwest state of India, provides an ideal ecosystem for the propagation of both the mosquito vectors (Aedes albopictus and Aedes aegypti) of dengue transmission.
Clinical manifestations may range from asymptomatic patients to Dengue Shock Syndrome. Many factors contribute to a consideration of bleeding in dengue infection, including the criteria for the grade of bleeding. Marked thrombocytopenia (Platelet count $<50 \times 109$ / L or less) indicates clinical severity, and is associated with an increased incidence of spontaneous bleeding. The effective control of bleeding is important in the treatment of patients with DHF / DSS. Patients with bleeding receive platelet transfusion to treat these complications. Thus, the decision to transfuse platelets is based on an overall assessment by the physician, clinical bleeding, and the availability of platelet component.

Currently, there is no specific treatment for dengue. Close clinical monitoring and careful fluid therapy is the only way of management for those with severe dengue disease, i.e., dengue hemorrhagic fever (DHF) or dengue shock syndrome (DSS) $)^{2}$ It is crucial to know among patients with dengue, who will potentially progress to DHF / DSS in the most reliable, economical and fastest way, so as to prioritize limited resources. There is an increasing prevalence of secondary dengue infection in the community, so more chance of bleeding manifestations ${ }^{5}$ Identification of clinically significant bleeding is important for subsequent optimal case management in dengue patients. Our study objectives were to identify proportion of bleeding manifestations seen in dengue patients and clinical outcome between patients who developed clinically significant bleeding.

\section{METHODS}

This was a descriptive longitudinal study carried out on patients admitted with dengue in medical ward and ICU of Govt. Medical College, Ernakulum from January 2018 to December 2018. Sample size was calculated as 230 based on previous study6

Study began after obtaining permission from state board of medical research (SBMR) and institutional review board. Confirmed cases of dengue fever by dengue viral antigen NS1 by card test or the antibodies against dengue virus - IgM either by card test or ELISA were identified. Those patients (or bystanders - in case of sick patients) who had given consent and fulfilling the inclusion criteria were taken up for the study. Consent from parents / Guardians was taken in less than 18 year old. Performa was filled after clinical examination and routine laboratory investigations. Patient was followed up clinically during the hospital stay till discharge. During hospital stay, bleeding manifestations like petechiae, melena, epistaxis, haematuria, haemoptysis, hematemesis, bleeding gums, menorrhagia and conjunctival haemorrhages were collected using a proforma. Outcome variables like relieved or death, duration of hospital stay, any complication, and transfusions if needed were noted in the proforma.

\section{Inclusion Criteria}

1. More than 13 years

2. Dengue confirmed by NS1Ag by card test or IgM Dengue either by card test or ELISA. 


\section{Exclusion Criteria}

1. Patients with other bleeding disorders

2. Patients with cardiac / liver / respiratory diseases

3. Patients or bystanders (in case of sick patients) not giving consent for the study

\section{Sample Size}

According to a study conducted by Chen $\mathrm{C}-\mathrm{M}$, Chan $\mathrm{K}-\mathrm{S}$, Yu W - L, Cheng K - C, Chao H - C, Yeh C - Y, et al. (6) gastrointestinal bleeding present among dengue patients was $31.4 \%$. Based on this data and using the sample size formula $(\mathrm{Z} \alpha)^{2} \mathrm{x} \mathrm{pq} / \mathrm{d} 2$ the minimum sample size found to be 228 . It was rounded to 230 .

$\mathrm{Z}$ value of alpha error at $5 \%$, which is equal to 1.96

$\mathrm{P}=$ proportion from previous study $=31.4 \%$

$\mathrm{Q}=100-\mathrm{P}=68.6 \%$

$\mathrm{D}=$ absolute precision of 6

$\frac{1.96 \times 1.96 \times 31.4 \times 68.6}{6 \times 6}=228$

\section{Statistical Analysis}

Data was coded and entered in Microsoft excel spread sheet. Data was analysed using SPSS statistical software. Qualitative data like age, sex, clinical features, presence of bleeding manifestations was tested using chi-square test. Difference between Mean platelet values between the two groups (with bleeding manifestations and without bleeding manifestations) was tested using unpaired t test. Outcome was expressed as frequency and percentage (death and relieved).

\section{RESULTS}

Out of 230 patients studied, 162 were males and 68 were females. Dengue fever was seen more among males. In the present study, mean age was 36.89 with a standard deviation of 16.60 , median 34.50 and mode 20.00 . The most common age group affected was $21-30$ and $41-50$ yrs. (23\%). Bleeding manifestations were seen in 102 (44.3\%) patients. Various bleeding manifestations seen in this study population were petechiae (34\%), purpura (24.3), ecchymosis (19.1\%), melena (10.4\%), epistaxis (3.5\%), haematuria (4.8\%), haemoptysis (2.2\%), hematemesis (0.9\%), gum bleed (17.8 $\%)$ menorrhagia (10.9\%) and conjunctival haemorrhage $(9.1$ $\%)$. Other clinical symptoms that were commonly observed were fever, myalgia and headache (96\%, $71 \%$ and $37 \%$ respectively).

In the current study, 29 patients (12.6\%) had complications such as gastrointestinal (11.3\%), respiratory (9.6\%), cardiovascular (4.3\%) and neurological (3\%). Complications were more when the duration of fever lasted for 6 - 10 days. Among clinical parameters only fever was associated with bleeding manifestations and was statistically significant (P value 0.006) (Table 1). $34.3 \%$ presented with duration of 4 days of fever, $26 \%$ with 3 days of fever and 15.2 $\%$ with 5 days of fever. Average duration of fever on admission was 4.3 days.
Previous history of dengue fever was seen in 23 patients. Previous history of dengue was highly associated with bleeding manifestations and was statistically significant (P value $<0.0001)$.

There were 66 males and 36 females with bleeding manifestations. Bleeding manifestations were more common among males, but there was no statistically significant association (P value 0.089) (Table 2).

\begin{tabular}{|c|c|c|c|c|c|c|c|}
\hline & & \multicolumn{4}{|c|}{ Bleeding } & \multirow{2}{*}{ Total } & \multirow{2}{*}{ P Value } \\
\hline & & Yes & $\%$ & No & $\%$ & & \\
\hline \multirow{2}{*}{ Fever } & Yes & 102 & 46.15 & 119 & 53.84 & 221 & \multirow{3}{*}{0.006} \\
\hline & No & 0 & 0 & 9 & 100 & 9 & \\
\hline \multicolumn{2}{|c|}{ Total } & 102 & 44.34 & 128 & 55.65 & 230 & \\
\hline \multicolumn{8}{|c|}{ Table 1. Association of Fever and Bleeding Manifestations } \\
\hline
\end{tabular}

\begin{tabular}{|c|c|c|c|c|c|}
\hline \multicolumn{2}{|c|}{ Sex } & \multicolumn{2}{|c|}{ Bleeding } & \multirow[t]{2}{*}{ Total } & \multirow[t]{2}{*}{ P Value } \\
\hline \multirow{3}{*}{ Male } & & Yes & No & & \\
\hline & Nos. & 66 & 96 & 162 & \multirow{4}{*}{0.089} \\
\hline & $\%$ & 40.7 & 59.3 & & \\
\hline \multirow{2}{*}{ Female } & Nos. & 36 & 32 & 66 & \\
\hline & $\%$ & 54.5 & 45.5 & & \\
\hline
\end{tabular}

\begin{tabular}{|c|c|c|c|}
\hline Sl No & Test & Frequency & $\%$ \\
\hline 1 & Igg & 35 & 15.2 \\
\hline 2 & Igm & 134 & 58.3 \\
\hline 3 & NS1 & 152 & 66.1 \\
\hline 4 & Igm \& NS1 & 63 & 27.4 \\
\hline \multicolumn{4}{|c|}{ Table 3. Lab Parameters } \\
\hline
\end{tabular}

\begin{tabular}{|c|c|c|c|c|c|c|c|}
\hline & & \multicolumn{4}{|c|}{ Bleeding } & \multirow{2}{*}{ Total } & \multirow{2}{*}{ P Value } \\
\hline & & Yes & $\%$ & No & $\%$ & & \\
\hline \multirow{2}{*}{ NS1 } & Positive & 58 & 38.15 & 94 & 61.84 & 152 & \multirow{3}{*}{0.008} \\
\hline & Negative & 44 & 56.41 & 34 & 43.58 & 78 & \\
\hline \multicolumn{2}{|c|}{ Total } & 102 & 44.34 & 128 & 55.65 & 230 & \\
\hline \multicolumn{8}{|c|}{ Table 4. Association of Bleeding Manifestation and NS1 Positive } \\
\hline
\end{tabular}

\begin{tabular}{|ccccc|}
\hline Sl.No & Variables & $\begin{array}{c}\text { With Bleeding } \\
\text { (Mean } \pm \text { SD) }\end{array}$ & $\begin{array}{c}\text { Without Bleeding } \\
\text { (Mean } \pm \text { SD) }\end{array}$ & $\begin{array}{c}\text { T Test } \\
\text { P-Value }\end{array}$ \\
\hline 1 & $\begin{array}{c}\text { Platelet count on } \\
\text { day 1 }\end{array}$ & $\begin{array}{c}39881.67 \pm \\
30544.33\end{array}$ & $79850.78 \pm 41242.25$ & 0.0001 \\
\hline \multirow{4}{*}{$\begin{array}{c}\text { Lowest Platelet } \\
16797.06 \pm \\
12072.88\end{array}$} & $53006.09 \pm 28096.47$ & 0.0001 \\
\hline \multicolumn{4}{|c}{ Table 5. Association of Bleeding Manifestation and } \\
Platelet Count on Day 1 and Dip in Platelet Count
\end{tabular}

The current study showed that, the serologic results for dengue fever was positive for NS1 antigen in 152 (66\%) patients, IgM antibodies positive in 134 (58 \%) patients and IgG antibody in 35 (15\%) patients. (Table 7).

Bleeding manifestations were seen more among patients with Dengue NS1Ag and IgG positive patients and was statistically significant. There was no significant association between bleeding manifestation and IgM positivity (Table 4 5).

Platelet count on first day of admission and the lowest platelet count values were associated with bleeding and were statistically significant $(\mathrm{P}<0.05)$ (Table 5). Platelet count on the first day of admission was associated with bleeding manifestations like petechia, purpura, ecchymosis, melaena, epistaxis, Gum bleed, menorrhagia, conjunctival haemorrhage and was statistically significant $(\mathrm{P}<0.05)$.

But it was not associated with haemoptysis, haematemesis, and haematuria $(\mathrm{P}>0.05)$. Lowest platelet count was associated with bleeding manifestations like petechia, purpura, ecchymosis, melaena, epistaxis, Gum bleed, menorrhagia, haemoptysis, conjunctival haemorrhage and was statistically significant $(\mathrm{P}<0.05)$ 
But it was not associated with haematemesis, haematuria (P $>0.05$ )

\section{DISCUSSION}

In the present study the total number of cases were 230 , out of which there were $162(70.4 \%)$ males and 68 (29.6\%) females. Male preponderance was seen in this study. It was similar to a study conducted by Senthil Kumar $\mathrm{K}$ et al. in Kerala, where $63.6 \%$ of the patients were males. ${ }^{9}$ There were 36 females and 66 males with bleeding manifestations. While, in the study conducted by Fernanda A Angerami et al. reported that $26 \%$ males and $18.6 \%$ females had bleeding manifestations. ${ }^{10}$ There was no association between gender and bleeding manifestations in the present study, though we found a male preponderance; probably due to more involvement of males in outdoor activities, with increased risk of exposure to mosquitoes. These observations were similar to a study conducted by Nishantkumar Pravinbhai Rathod et al. in Mumbai. ${ }^{11}$

Most common age group affected in this study population was $21-30$ and $41-50$ yrs. (23\%), and mean age was 36.89 . Similarly, in a recent study done by Rajoo et al. Mean age was 31.6 years with a range of 15 to 80 years, with predominant male patients (male:female ratio $=3.3: 1$ ) ${ }^{12}$ Bleeding manifestations were found to be common in the age group of 21 - 30 years and $41-50$ years. It was almost similar to a study conducted by Anish Laul, Poonam Merugumala et al. in New Delhi, India, in which it was reported that the incidence was common in age group of $21-30$ years. ${ }^{13}$ In the current study, 23 cases had previous history of dengue fever. It was highly associated with bleeding manifestations (95.65 \%) and was statistically significant ( $P$ value 0.0001 ). It was similar to a study conducted by Thomas et al. ${ }^{14}$ which reported bleeding manifestations and severe illness was more frequent in patients with secondary dengue infection (odds ratio, 7.18; $95 \%$ confidence interval, $\mathrm{P}<0.001$ ).

The current study showed that, the serologic results for dengue fever was positive for NS1 antigen in 152 (66\%) patients, IgM antibodies positive in 134 (58\%) patients and IgG antibody (15\%). It was similar to a study conducted by M.V.S. Subbalakshmi et al. in Kerala where NS1 antigen was detected in $80(54.1 \%)$ patients, IgM antibodies positive in $101(68.2 \%)$ patients and IgG antibody (26.4\%). ${ }^{15}$ The most common symptoms noticed were fever $96 \%$ followed by Myalgia in $71 \%$ and headache in $37 \%$. It was similar to a study conducted by Senthil Kumar K. et al. where the most common symptoms noticed were fever $93 \%$ followed by headache in $36 \%$ and myalgia in $15 \% .{ }^{9}$

In the present study, the duration of fever ranged from 4 to 5 days and the mean delay in admission after appearance of fever was 5.1 days. In a study conducted by Jacob k Jacob et al. the mean duration of fever on presentation to the hospital was $3.23 \pm 0.649$ days $^{16}$ Bleeding manifestations seen in this study population were $44.3 \%$, where as in a study conducted by Bashir $\mathrm{AB}$ et al. bleeding manifestations were $10.5 \%{ }^{17}$ Another study conducted by A. H. Fagbami, P J. U. Mataika, et al. among the adults hospitalized for dengue, $43 \%$ had haemorrhagic manifestations, including epistaxis, gingival bleeding, haematemesis, melaena and haematuria. ${ }^{18}$
Various bleeding manifestations seen in this study population were petechiae (34\%), purpura (24.3), ecchymosis (19.1\%), melena (10.4\%), epistaxis (3.5\%), haematuria (4.8\%), haemoptysis (2.2\%), hematemesis $(0.9$ $\%)$, gum bleed (17.8\%), menorrhagia (10.9\%) and conjunctival haemorrhage $(9.1 \%)$ of patients. None of the patients had intracranial bleeding. In a study conducted by S. Sharma, S. K Sharma et al. the haemorrhagic manifestations included erythematous macular skin rash $(36.7 \%)$ and extensive ecchymoses (19.4\%). Haematemesis occurred in $22.4 \%$ and melena occurred in $26.5 \%$ of the patients. In 10.2 $\%$ of the patients both haematemesis and melena occurred. Epistaxis was noted in $32.6 \%$ of the patients. ${ }^{19}$

Conjunctival haemorrhage was seen in only $9.1 \%$ of our study population, but it was less when compared with a similar study conducted by Ritu Karoli, Jalees Fatima, et al.20 In sexually active women, dengue can co-present with menstruation and this can lead to severe dysmenorrhea and excessive menstrual bleeding. In our study, $10.9 \%$ of women had menorrhagia, but the results were different when compared to study conducted by Kanchana Tangnararatchakit et al. where $29 \%$ had vaginal bleeding. ${ }^{21}$

In the current study, 29 patients (12.6\%) had complications related to gastrointestinal (11.3\%), respiratory $(9.6 \%)$, cardiovascular $(4.3 \%)$ and neurological (3\%). However no mortality was observed in the present study. It was relatively similar to a study, conducted by Anish Laul, et al. where neurological involvement was seen in $2.6 \%$ patients. ${ }^{13}$ and a study conducted by Yacoub et al. where cardiovascular complications were seen in $4 \% .22$ In this study, $9.6 \%$ cases had respiratory complications, but a similar study conducted by Mohamed et al. showed that the results were different, where respiratory complications were seen in $23 \%$ of cases. ${ }^{23}$

\section{CONCLUSIONS}

In the present study out of 230 subjects, 162 subjects were males $(70.4 \%)$ and 68 were females $(29.6 \%)$. The mean age was 36.89 with a standard deviation of 16.60 . The most common age groups affected were $21-30$ and $41-50$ yrs. (23\%). $44.3 \%$ had bleeding manifestations with significant association between factors such as age, fever and IgG positivity. A low platelet count on admission especially less than 10,000 was significantly associated with these bleeding manifestations. It was observed that major complications were involvement of gastrointestinal and respiratory system. These significant observations are important for both the primary care and tertiary care physicians in meticulous management and referral of dengue patients.

\section{Limitations of the Study}

- The study period was for one year only.

- The actual picture of dengue cases and those with bleeding manifestations were underestimated because only patients referred to the GMC, Ernakulam during the particular study period were included in the study

- Being a tertiary care center in metro city, study might not always represent actual situation in the community. 
- There was no mortality in the cases studied because the study was done in a tertiary care center with adequate facilities.

- We did not test the serotype of dengue viruses.

- Dengue fever cases were less when compared to the previous years.

Data sharing statement provided by the authors is available with the full text of this article at jemds.com.

Financial or other competing interests: None.

Disclosure forms provided by the authors are available with the full text of this article at jemds.com.

\section{REFERENCES}

[1] Schaefer TJ, Panda PK, Wolford RW. Dengue fever. In: StatPearls. Treasure Island (FL): StatPearls Publishing 2021 Jan. [Updated 2020 Aug 23]. https://www.ncbi.nlm.nih.gov/books/NBK430732/

[2] Government of India. Guidelines for clinical management of dengue fever, dengue haemorrhagic fever and dengue shock syndrome. Directorate of NVBDCP 2008: p. 33.

[3] Cavinato JL. CCCC. Supply chain and transportation dictionary. US: Springer 2000:49-84.

[4] Bhaskar S, Ilanthodi S, Rao PS, et al.. Pattern analysis of thrombocytopenia in dengue versus non-dengue patients. National Journal of Laboratory Medicine 2018;7(4):PO23-7.

[5] Sanyaolu A, Okorie C, Badaru 0, et al.. Global epidemiology of dengue hemorrhagic fever: an update. J Hum Virol Retrovirology 2017;5(6):00179.

[6] Cogan JE. Dengue and severe dengue. WHO 2018:1-7. http://www.who.int/en/news-room/factsheets/detail/dengue-and-severe-dengue

[7] WHO. Dengue and severe dengue. Vol. 117. World Health Organisation Fact Sheet 2014:1-4. www.who.int/mediacentre/factsheets/fs117/en/index. html

[8] Murhekar M, Joshua V, Kanagasabai K, et al.. Epidemiology of dengue fever in India, based on laboratory surveillance data, 2014-2017. Int J Infect Dis 2019;84S:S10-4.

[9] Kumar KS, Rajendran NK, Brabhukumar CA. Clinical profile of dengue fever in children: analysis of 2017 outbreak from Central Kerala, India. Int J Contemp Pediatr 2018;5(6):2265-9.
[10] Orsi FA, Angerami RN, Mazetto BM, et al.. Reduced thrombin formation and excessive fibrinolysis are associated with bleeding complications in patients with dengue fever: a case-control study comparing dengue fever patients with and without bleeding manifestations. BMC Infect Dis 2013;13(1):350.

[11] Rathod NP, Ansari NJR, Singh DK. Clinical profile of children with dengue and factors associated with severe dengue and dengue with warning signs. Pediatr Oncall 2018;15(1):1-4.

[12] Jnaneshwari M, Jayakumar S, Kumar A, et al.. Study of serum aminotransferase levels in dengue fever. J Evol Med Dent Sci 2014;3(10):2445-55.

[13] Laul A, Laul P, Merugumala V, et al.. Clinical profiles of dengue infection during an outbreak in Northern India. J Trop Med 2016;2016:5917934.

[14] Thomas M, Kumar NP. Microevolutionary trends of dengue virus-3 in Kerala, India. Indian J Exp Biol 2015;53(4):236-40.

[15] Subbalaxmi MVS, Kumar GV, Ramanjaneyulu KH, et al. Clinical, laboratory profile and outcome of dengue fever at a south Indian tertiary care hospital. J Clin Sci Res 2017;6(3):160-9.

[16] Jacob JK, George SB, Anandkumar A, et al.. Regional clinical profile and predictors of thrombocytopenia in adults with dengue fever. International Journal of Scientific Study 2016;4(6):7-18.

[17] Bashir AB, Mohammed BA, Saeed OK, et al.. Thrombocytopenia and bleeding manifestations among patients with dengue virus infection in Port Sudan, Red Sea State of Sudan. J Infect Dis Immun 2015;7(2):7-13.

[18] Fagbami AH, Mataika JU, Shrestha M, et al.. Dengue type 1 epidemic with haemorrhagic manifestations in Fiji, 1989-90. Bull World Health Organ 1995;73(3):291-7.

[19] Sharma S, Sharma S, Mohan A, et al.. Clinical profile of dengue haemorrhagic fever in adults during 1996outbreak in Delhi, India. Dengue Bulletin 1998;22:20-30.

[20] Karoli R, Fatima J, Siddiqi Z, et al.. Clinical profile of dengue infection at a teaching hospital in North India. J Infect Dev Ctries 2012;6(7):551-4.

[21] Ganguli SG. Unusual cases of malaria. Antiseptic 1947;44(7):470.

[22] Yacoub S, Wertheim H, Simmons CP, et al.. Cardiovascular manifestations of the emerging dengue pandemic. Nat Rev Cardiol 2014;11(6):335-45.

[23] Mohamed NA, El-Raoof EA, Ibraheem HA. Respiratory manifestations of dengue fever in Taiz-Yemen. Egypt J Chest Dis Tuberc 2013;62(2):319-23. 